\title{
Catatonic syndrome - positive and differential diagnostic controversies. Case study
}

\author{
Diana Cătălina Aniculăesei, Dania-Andreea Radu, \\ Alexandra Boloș, Ilinca Untu, Roxana Chiriță
}

Diana Cătălina Aniculăesei - M.D., "Socola” Institute of Psychiatry, Iaşi, Romania Dania Andreea Radu - M.D., Ph.D. Student, UMF "Grigore T. Popa”, Iași, "Socola” Institute of Psychiatry, Iaşi, Romania

Alexandra Boloș- M.D., Ph.D., UMF “Grigore T. Popa”, Iași, “Socola” Institute of Psychiatry, Iaşi, Romania

Ilinca Untu - M.D., Ph.D. Student, UMF “Grigore T. Popa”, Iaşi, “Socola” Institute of Psychiatry, Iaşi, Romania

Roxana Chiriță - Prof., M.D., Ph.D., UMF "Grigore T. Popa", Iași, "Socola" Institute of Psychiatry, Iaşi, Romania

\begin{abstract}
The present paper is the presentation of a case that exposes a positive and differential diagnosis controversy, in the context of a catatonic syndrome with a sudden onset, occurring in an apparent state of mental health, in a patient of 42 years old, without personal or heredocolateral psychiatric history, with normal premorbid functioning. Its purpose is to emphasize the importance of excluding organicity in the case of catatonic syndrome and to make it aware that the impact of the often impressive psychotic symptoms should not overshadow the detection of potentially life-threatening somatic symptoms. The presented case underlines precisely the dilemma of establishing the etiology of catatonic syndrome, between endogenous causes and underdiagnosed meningeal reaction.
\end{abstract}

\section{KEY WORDS:}

Catatonia, meningeal reaction, organicity, differential diagnosis

Catatonic syndrome is mainly characterized by motor symptoms that can occur in psychiatric context as well as in catatonic schizophrenia and in a wide variety of organic disorders, hence the fundamental importance of making a rigorous differential diagnosis and the diagnostic dilemma of the present case. Catatonia is not a mental disorder per se, but a syndrome associated with various somatic or psychiatric conditions $(1,2)$. 
The components of this syndrome can be systematized into two categories:

- negative symptoms that involve the absence of motor activity or its marked reduction;

- positive symptoms that involve excessive and pathological growth of motor activity.

Positive symptoms include: bizarre postures of body segments maintained for extended intervals of time, catalepsy (lack of response to stimuli, slowing of movements until the occurrence of bizarre positions), mannerisms, stereotypes, catatonic agitation with aggressive behavior that may occur in complete catatonic state, suggestibility manifested by motor automatisms expressed as automatic acts of response to the surrounding demands, ecopraxia, ecolalia and ecomimia (known as the ecopathic triad) (1, $3,4)$.

The negative symptoms are presented by: catatonic stupor, negativism (resistance to the request to execute certain demands, to answer the interlocutor's questions), mute (the complete absence of any verbal emission), ambitendence (contrary tendencies that appear to be in conflict) $(4,5)$.

Etiological factors of catatonic syndrome can be:

Psychiatric disorders

- Schizophrenia;

- Schizoaffective disorder;

- Delusional disorder;

- Mood disorders (Major depression);

Cerebrovascular diseases

- Aneurysm of the anterior cerebral artery;

- Thrombosis;

- Bleeding;

- Temporal epilepsy

Degenerative diseases

- Parkinson's disease;

- Huntington's Disease;

- Cerebral atrophy;
- Wilson's Disease;

Endocrine-metabolic diseases and tumoral diseases

- Diabetic ketoacidosis;

- Neoplasia;

- Wernicke's encephalopathy;

Alcoholism / Drugs

- Acute alcoholic intoxication;

- Alcohol impregnation;

- Amphetamines;

- Barbituric seizure;

- Cocaine;

- Alkaloids of Belladonna;

Other medical conditions

- AIDS;

- Vitamin B12 deficiency;

- CO poisoning;

- Neurosyphilis;

- Systemic erythematous lupus.

\section{THE REASONS OF THE PSYCHIATRIC ADMISSION}

A 42-year-old male patient from the urban area, at the first admission at the Psychiatric Institute "Socola" Iasi, presented as a psychiatric emergency, brought by his brother, for a symptomatology with a sudden onset with approximately one week ago, manifested by food and water refusal, verbal negativism, psychomotor inhibition, hallucinatory behavior. In addition to psychiatric symptoms, it presents mild photophobia, skin hyperesthesia, ataxic gait and generalized fine tremor whose onset is approximately three days prior to admission.

\section{DEMOGRAPHIC AND PERSONAL}

DATA: in terms of socio-family and professional insertion, he has an average level of education, currently does not work, is unmarried, has no children and lives with his brother. 
PATHOLOGICAL AND HEREDOCOLATERAL PERSONAL BACKGROUND: The patient does not have a personal history of psychiatric disorders nor psychiatric relevant heredocolateral history for first-degree and second-degree relatives.

\section{HISTORY OF THE DISEASE}

The patient, who was first admitted to the Psychiatry Institute "Socola" Iaşi, without psychiatric records in other counties, with a good premorbid functioning, is brought with the ambulance, after being consulted previously in the Neurology and Infectious Diseases service for the diagnosis of exclusion of an organic catatonic syndrome. The aspects of the cranio-cerebral CT of the Clinic of Neurology were presented within normal limits, without suggestive elements for an acute neurological process.

The clinical examination performed at the Infectious Diseases Hospital prior to hospitalization revealed the following: afebrile patient, without meningeal contracture syndrome, normal stetacoustic cardio-pulmonary, catatonic. The patient was referred to the Psychiatric Clinic with the recommendation of an interclinical reevaluation the following day, in order to perform the lumbar puncture for differential diagnosis and recommendation to initiate antibiotic treatment with Cefotaxim $4 \mathrm{~g}$ /day (2g/12h).

The second consultation in the Clinic of Infectious Diseases no longer required the lumbar puncture, establishing a catatonic symptomatology, but without signs of meningeal contracture, intracranial hypertension syndrome or inflammatory biological syndrome, without clinicobiological data suggestive for meningitis, fact for which the patient returned to the Psychiatric Clinic in order to continue hospitalization.
PSYCHOLOGICAL EXAMINATION reveals: Dysthymic configuration with psychotic manifestations/negative picture, anhedonic-developmental and severe integrative-relational deficit, food and verbal negativism.

\section{PSYCHIATRIC}

EXAMINATION

highlights: Patient apparently calm, with psycho-motor inhibition, disoriented temporospatial, in state of stupor/ marked psychomotor inhibition, catatonic, non-cooperative, non-communicative, presents verbal negativism, refusal of water and food, athymia, apparent hallucinatory behavior that remits after about 3-4 days, immobile mimic, inexpressive, does not initiate eye contact with the examiner and cannot provide information. It presents a severe integrativerelational socio-familial deficit, in a catatonic context of unspecified etiology.

\section{CLINICAL EXAM:}

- marked psycho-motor inhibition;

- state of nutrition - normoponderal;

- pale skin and mucous, subcutaneous tissue normally represented;

- active opposition in opening the eyes, photophobia, skin hyperesthesia, posturing, without waxy flexibility;

- normal stetacoustic lung, rhythmic cardiac noises.

\section{PARACLINICAL EXPLORATIONS:}

HLG, VSH, urea, serum creatinine, blood glucose, TGO, TGP, GGT, Na, Cl, K performed in the Neurology Clinic and in the Psychiatry Clinic at the hospital did not show any significant changes - except for mild thrombocytopenia - thrombocytopenia - $131 \mathrm{x}$ $103 / \mu \mathrm{L}$ (abnormal value $150-400 \times 103 /$ $\mu \mathrm{L})$, low serum bicarbonate - $16 \mathrm{mmol} / \mathrm{l}$ (normal values 22-30 mmol/l), respectively CK-MB increased - $20 \mathrm{U} / \mathrm{L}$ (abnormal value 0 $-16 \mathrm{U} / \mathrm{L})$. 
on

Has not submitted EKG changes; the EEG exam revealed: Spontaneous path with dominant alpha-hypovolated rhythm, slow, not influenced by eye opening.

One week after admission, the patient undergoing antibiotic therapy initiated at the medical recommendations of the Infectious Diseases Clinic, presents with paroxysmal, isolated $\left(38^{\circ} \mathrm{C}\right)$ febrile episodes, respectively episodes of subfebrility, skin hyperesthesia and photophobia, modification of hematological samples of mild type pancytopenia, requiring a third consultation in the Infectious Diseases Clinic, after which a meningeal irritation syndrome is excluded, the patient being referred to the Psychiatry Clinic, with the recommendations of association to the initial therapeutic scheme of $500 \mathrm{mg}$ of Ciprofloxacin at 12 hours.

At three weeks after admission, the craniocerebral CT is repeated because the catatonic elements persist despite the specific psychotropic treatment.

The cranio-cerebral examination reveals: Discrete high frontal cerebral atrophy, bilaterally symmetrical, without CT elements suggestive for subarachnoid hemorrhage, intra- or extra-nevrachial hematomas, ischemic stroke or expansive brain formation on the native sections.

Following the corroboration of the clinicoanamnestic data with the psychiatric and psychological examination, the diagnosis of Acute Psychotic Disorder with catatonic elements was established.

\section{TREATMENT}

From a therapeutic point of view, in addition to the prophylactic antibiotherapy initiated according to the protocols, according to the recommendations, the patient has undergone psychotropic treatment with atypical antipsychotics according to the scheme: Olanzapine $5 \mathrm{mg}$ in the evening, with the dose increased to $10 \mathrm{mg} / \mathrm{day}$, in the evening,
Bromazepam 1.5 mgx 3 tablets daily, in the morning, lunch and evening, Diazepam an ampoule i.m. if needed and SG 10\% $500 \mathrm{ml}+$ vit B1 + vit B6 i.v.. One week after admission, Olanzapine was discontinued and a vial of Aripiprazole $9.75 \mathrm{mg}$ /day was introduced, intramuscularly, in the morning, the combination of hypnotics Zolpidemum 10mgx 3cp/day, tricyclic antidepressants Clomipramine hydrochloride $25 \mathrm{mgx} 3 \mathrm{cp} /$ day, antibiotic therapy and benzodiazepine cessation. Due to the lack of therapeutic response, one week later, Aripiprazole is replaced with Olanzapinum $15 \mathrm{mg}$, in the evening, Piracetam $1 \mathrm{~g} / 5 \mathrm{ml}$ one ampoule /day is associated with maintaining the rest of the therapeutic regimen. Two weeks after hospitalization, the antibiotic therapy is stopped, maintaining the psychotropic medication according to the schedule, respectively the increase of Olanzapine at $20 \mathrm{mg} /$ day - (discrete impregnation elements, with the introduction of a tablet of trihexifenidyl hydrochloride $2 \mathrm{mg}$, in the evening, respectively the increase of 2 per day thereafter) and increasing the dose of clomipramine hydrochloride to 4 doses of 25 $\mathrm{mg} /$ day. Following symptoms remission, 6 weeks after hospitalization, Zolpidemum doses gradually decreased until discontinuation, and the patient was maintained on the following regimen: Clomipramine Hydrochloride $25 \mathrm{mgx} 3 \mathrm{cp} /$ day (1-1-1), Trihexiphenidyl Hydrochloride 2mgx 2c /day (1-0-1), Piracetam 600mgx1cp /day, morning (1-0-0) and Olanzapine 20mgx 1cp /day, evening (0-0-1).

\section{EVOLUTION}

During the hospitalization period, the symptoms gradually recovered, the patient mobilizing alone, becoming cooperative, communicative, with the remission of the symptoms of hyperesthesia, photophobia, posturing, water and food refusal. At fallow- 
ндн

up, one month after discharge, the patient

presents favorable evolution, with no psychiatric symptoms.

\section{DISCUSSION}

Catatonic syndrome is characterized by abnormal motor behaviors and can be encountered both in psychiatric context and in a wide variety of organic pathologies, hence the importance of differential diagnosis and the diagnostic dilemma of the case $(1,2)$. The diagnostic dilemma is represented by the origin of catatonic syndrome (organic or endogenous) due to the overlap of the somatic and psychiatric symptoms. The gradual remission of symptoms occurred after two weeks, concomitantly with the cessation of antibiotic therapy, with the absence of any recurrence so far, which may suggest an underlying organic disorder (atypical meningeal syndrome). Also, the problematic of the diagnosis also puts pressure on the therapeutic conduct, the patient being subjected to a complex therapeutic association, after which the remission was obtained.

In the literature, Zolpidem has shown its role in improving the symptoms of catatonia, with numerous studies mentioning it $(6,7,8,11$, $12,13)$, thus leading to the creation of a "Response test" therapeutically" $(9,10)$.

\section{CONCLUSIONS}

Careful monitoring and examination of the patient is very important to rule out an organic catatonic syndrome. The therapeutic response was obtained with the help of a constant therapeutic regimen adapted to the patient's condition, an important role in the psycho-motor disinhibition with Zolpidem and Clomipramine. The patient could have suffered from an organic disorder with atypical symptoms that was resolved with the help of antibiotics, considering his age for the manifestation of a first psychotic episode.

\section{ACKNOWLEDGEMENTS AND DISCLOSURES}

The authors declare that they have no potential conflicts of interest to disclose.

\section{REFERENCES}

1. 1 Prelipceanu, D. 2015. Psihiatrie clinică. Bucureşti: Editura Medicală

2. Appiani, F. J., Castro, G. S.2018. Catatonia is not schizophrenia and it is treatable. Schizophrenia research, 200, 112-116.

3. Bartolommei, N., Lattanzi, L., Callari, A., Cosentino, L., Luchini, F., Mauri, M. 2012. Catatonia: a critical review and therapeutic recommendations. Journal of Psychopathology, 18, 234-246.

4. Francis, A. 2010. Catatonia: diagnosis, classification, and treatment. Current psychiatry reports, 12(3), $180-185$.

5. Hlal, H., Kettani, N., Berhili, N., Rammouz, I., Aalouane, R. 2014. Place du zolpidem dans le traitement des catatonies résistantes aux benzodiazépines. À propos d'un cas. La Presse médicale, 43(9), 1018-1020.

6. Javelot, H., Michel, B., Steiner, R., Javelot, T., Cottencin, O. 2015. Zolpidem test and catatonia. Journal of clinical pharmacy and therapeutics, 40(6), 699-701.

7. Peglow, S., Prem, V., McDaniel, W. 2013. Treatment of catatonia with zolpidem. The Journal of neuropsychiatry and clinical neurosciences, 25(3), E13-E13.

8. Sienaert, P., Dhossche, D. M., Vancampfort, D., De Hert, M., Gazdag, G. 2014. A clinical review of the treatment of catatonia. Frontiers in psychiatry, 5, 181.

9. Thomas, P., Rascle, C., Mastain, B., Maron, M., Vaiva, G. 1997. Test for catatonia with zolpidem. The Lancet, 349(9053), 702. 
110/Bulletin of Integrative Psychiatry ONew Series O September 2019 ○ Year XXV ○ No. 3 (82)

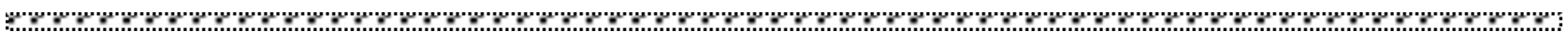
10. Cottencin, O., Warembourg, F., de Lenclave, M. B. D. C., Lucas, B., Vaiva, G., Goudemand, M., Thomas, P. 2007. Catatonia and consultation-liaison psychiatry study of 12 cases. Progress in Neuro-Psychopharmacology and Biological Psychiatry, 31(6), 1170-1176.

11. http://www.jpsychopathol.it/issues/2012/vol18-3/05Bartolommei.pdf

12. https://www.frontiersin.org/articles/10.3389/fpsyt.2014.00181/full

https://www.researchgate.net/publication/283577105_Zolpidem_test_and_catatonia

\section{Correspondence:}

Ilinca Untu,

M.D., Ph.D. Student, UMF “Grigore T. Popa”, Iași, “Socola” Institute of Psychiatry, Iaşi, Romania, ilincauntu21@gmail.com

Submission: 16 july 2019

Acceptance: 29 aug 2019 